\title{
CHARITÉ
}

\section{CLOVER-DBS: Algorithm-Guided Deep Brain Stimulation-Programming Based on External Sensor Feedback Evaluated in a Prospective, Randomized, Crossover, Double-Blind, Two-Center Study}

Gregor R. Wenzel, Jan Roediger, Christof Brücke, Ana Luísa de A. Marcelino, Eileen Gülke, Monika Pötter-Nerger, Heleen Scholtes, Kenny Wynants, León M. Juárez Paz, Andrea A. Kühn

\section{Document type}

Postprint (accepted version)

This version is available at

https://doi.org/10.17169/refubium-31024

\section{Citation details}

Wenzel GR, Roediger J, Brücke C, Marcelino AL de A, Gülke E, Pötter-Nerger M, et al.

CLOVER-DBS: Algorithm-Guided Deep Brain Stimulation-Programming Based on External Sensor Feedback Evaluated in a Prospective, Randomized, Crossover, Double-Blind, Two-Center Study. Journal of Parkinson's Disease. [Online] NL: IOS Press; 2021; 1-13. DOI: 10.3233/JPD-202480

Terms of use

All rights reserved. This document is intended solely for personal, non-commercial use.

The final publication is available at IOS Press through http://dx.doi.org/10.3233/JPD-202480. 


\title{
CLOVER-DBS - Algorithm-guided DBS-programming based on external sensor feedback evaluated in a prospective, randomized, crossover, double-blind, two-center study
}

\author{
Gregor R. Wenzel ${ }^{a}$, Jan Roediger ${ }^{a, b}$, Christof Brücke ${ }^{a}$, Ana Luísa de A. Marcelino ${ }^{a}$, Eileen Gülke ${ }^{c}$, \\ Monika Pötter-Nerger ${ }^{c}$, Heleen Scholtes ${ }^{d}$, Kenny Wynants ${ }^{d}$, León M. Juárez Paz ${ }^{d}$, and Andrea A. Kühn ${ }^{a}$ \\ a) Department of Neurology, Movement disorders \& Neuromodulation section, Charité - \\ University Medicine Berlin, Germany \\ b) Einstein Center for Neurosciences Berlin, Charité - University Medicine Berlin, Germany \\ c) Department of Neurology, Universitätsklinikum Hamburg-Eppendorf, Germany \\ d) Boston Scientific, Valencia, California
}

This article has been published in the Journal Of Parkinson's Disease on June 12, 2021 and is available from https://content.iospress.com/openurl?genre=article\&id=doi:10.3233/JPD-202480

\begin{abstract}
Background

Recent technological advances in deep brain stimulation (DBS) (e.g., directional leads, multiple independent current sources) lead to increasing DBS-optimization burden. Techniques to streamline and facilitate programming could leverage these innovations.
\end{abstract}

Objectives

We evaluated clinical effectiveness of algorithm-guided DBS-programming based on wearable-sensorfeedback compared to standard-of-care DBS-settings in a prospective, randomized, crossover, doubleblind study in two German DBS centers.

Methods

For 23 Parkinson's Disease patients with clinically effective DBS, new algorithm-guided DBS-settings were determined and compared to previously established standard-of-care DBS-settings using UPDRS-III and motion-sensor-assessment. Clinical and imaging data with lead-localizations were analyzed to evaluate characteristics of algorithm-derived programming compared to standard-of-care. Six different versions of the algorithm were evaluated during the study and 10 subjects programmed with uniform algorithm-version were analyzed as a subgroup. 
Results

Algorithm-guided and standard-of-care DBS-settings effectively reduced motor symptoms compared to off-stimulation-state. UPDRS-III scores were reduced significantly more with standard-of-care settings as compared to algorithm-guided programming with heterogenous algorithm versions in the entire cohort. A subgroup with the latest algorithm version showed no significant differences in UPDRS-III achieved by the two programming-methods. Comparing active contacts in standard-of-care and algorithm-guided DBS-settings, contacts in the latter had larger location variability and were farther away from a literature-based optimal stimulation target.

\section{Conclusions}

Algorithm-guided programming may be a reasonable approach to replace monopolar review, enable less trained health-professionals to achieve satisfactory DBS-programming results, or potentially reduce time needed for programming. Larger studies and further improvements of algorithm-guided programming are needed to confirm these results. 


\section{Introduction}

Deep brain stimulation (DBS) has become a standard treatment option for advanced Parkinson's disease (PD)[1,2]. With stimulation of the subthalamic nucleus (STN) as one of the most widely used target, about $50-60 \%$ improvement in motor symptoms can be achieved[3,4]. A recent review found the optimal target region to be central to dorsolateral within the STN[5]. Usual clinical assessment after DBS-implant includes a so-called monopolar review to test each contact on the lead for clinical effect and side effects. The development of directional DBS leads with segmented (i.e., directional), tripartite contacts offers the option to preferentially stimulate regions in a particular direction perpendicular to the lead's axis[6]. The main aim of this technical innovation is to optimize treatment when lead placement has been suboptimal, i.e., rendering the current towards the optimal targetregion such as the dorsolateral/motor STN in PD while avoiding regions related to side effects $[6,7]$. Recent studies indicate an enlarged therapeutic window $[7,8]$ and reduced side effects $[9,10]$ for directional leads. However, in clinical practice, the tremendous increase in possible contact configurations is an unsolved problem that makes it virtually impossible to cover every possibility in a monopolar review[6], which currently requires a highly trained person[11-14] and still remains the first crucial step in management of DBS-patients. Therefore, strategies are needed to replace the full monopolar review by a semi-automated procedure to identify optimal contact configurations for the further DBS-optimization process. Motor symptom assessment by wearable sensors is increasingly used as a relatively objective, examiner-independent measure and is even discussed to potentially replace clinical evaluation as a gold standard in the future $[2,11,15,16]$. This study evaluates a semiautomatic iterative-loop algorithm using motion-sensor feedback to determine optimal contact selection and stimulation amplitude in patients treated with STN-DBS.

\section{Subjects and Methods}

\section{Study design}

CLOVER-DBS is a prospective and multicenter study with a randomized, crossover and double-blind design. Because of the exploratory nature of the study, no primary endpoint was defined. The clinically most important exploratory endpoint of the study is DBS-induced improvements in full UPDRS-III scores yielded by computer-algorithm-guided DBS-programming (AgP) compared to standard-of-care (SoC) DBS-settings. During the study visits always the full UPDRS-III was recorded during the assessments. Some analyses in this manuscript required a hemibody UPDRS-III, which is specified in the respective text passage. Further exploratory per-protocol endpoints of the study compare bradykinesia (assessed by an external finger mounted sensor (Kinesia One ${ }^{\mathrm{TM}}$, Great Lakes Neurotech, 
Cleveland, Ohio)) and rigidity scores (clinical assessment) yielded by AgP and SoC DBS-settings. The time and number of steps needed to reach AgP and SoC DBS-settings were intended as an exploratory endpoint in the study protocol. Additional exploratory endpoints added in the final analysis were: a) identification of relevant influencing factors for AgP effectiveness by a mixed-effects model; b) location analysis in Montreal Neurological Institute (MNI) space comparing active contacts selected in AgP and SoC DBS-setting. The study is registered as NCT03037398 at ClinicalTrials.gov.

\section{Study subjects}

Parkinson's Disease (PD) patients were recruited at two German centers (Charité - University Medicine Berlin $n=20$ and University Medical Center Hamburg-Eppendorf $n=3$ ). Both centers obtained ethics committee approval for the study and written informed consent was obtained from all subjects prior to study inclusion. Subjects had undergone on-label bilateral STN-DBS (Boston Scientific, Valencia, California; 8-ring $(n=3)$ or directional $(n=20)$ leads) for treatment of advanced PD at least 6 months before enrollment. DBS-programming was carried out according to standard clinical procedures in both centers prior to recruitment for the study. For inclusion in this study, subjects' pre-operative UPDRS-III score had to be $\geq 25$ points in off-medication-state including bradykinetic-rigid symptoms. Subjects with tremor-dominant PD-phenotype were not included as tremor was judged to be potentially less reliable compared to bradykinesia and rigidity. Furthermore, SoC DBS-settings had to be stable for at lead 4 weeks prior to enrollment, and yield $\geq 30 \%$ reduction in UPDRS-III scores in the off-medication-state. Finally, study candidates were excluded if their optimal stimulation settings included multiple frequencies or stimulation on non-adjacent contacts. All tests were performed in the off-medication-state, i.e., after $\geq 12$ hours of withdrawal of antiparkinsonian medication.

\section{Study protocol}

The study protocol is summarized in Fig. 1A. The Kinesia One ${ }^{\mathrm{TM}}$ sensor system rates the subitems speed, amplitude and rhythmicity of the task on a UPDRS-III-like scale from 0 to 4 by a proprietary signal processing software [17]. The median of all battery subitems was used for analysis.

First DBS was switched off for $\geq 30$ minutes for baseline assessment, which included full UPDRS-III and a sensor-based test battery (finger taps, hand grasps, resting tremor, all bilateral). DBS was then programmed to a unilateral test stimulation suggested by the algorithm. After 30 seconds wash-in time, bradykinesia was assessed by sensor measurement and rigidity by clinical rating on the contralateral side. Either speed or amplitude were used as input for the algorithm. The choice depended on results from previous subjects and (if available for the respective subject) change of the 
respective subitem (speed/amplitude) in baseline testing (off-stimulation-state vs. on-stimulationstate). Results were entered in the algorithm user interface and a next suggestion was calculated based on all previous results.

This loop was continued until predefined criteria for sufficiently effective algorithm-guided settings were achieved (see also Fig. 1C). After repeating the algorithm-cycle for the contralateral side, AgP and SoC DBS-settings were evaluated in randomized order by full UPDRS-III and the sensor-based test battery (see also Fig. 1D). For each of the tested DBS-settings, a 30 minutes wash-in time was allowed and both, the subject and the physician, were blinded to the DBS-settings being evaluated. Subjects and physician then consented the go-home DBS-setting (AgP or SoC). Time consumption for SoC programming was estimated retrospectively from charts and a focused medical history during the study visit.

\section{Generation of DBS-Setting-Suggestions by the Algorithm}

The algorithm is a standalone software that explores the clinical effect of different stimulation settings in an iterative loop by representing them in two-dimensional spaces (Fig. 2). Each point in the twodimensional space represents defined stimulation settings (i.e., contact configuration and stimulation amplitude). Dimensions are stimulation amplitude vs. one spatial dimension, which can be either the vertical position of the activated contacts for ring-mode or the angle of the activated contacts for directional mode stimulation. The algorithm explores these two-dimensional spaces based on a weighting method, whose weights were adjusted manually to improve its performance based on the results of study visits of previous subjects. The weighting method uses sensor-rated scores (e.g., finger tapping speed) yielded by each explored point as input to generate (or update) a score prediction map in the two-dimensional space.

The algorithm keeps frequency at $130 \mathrm{~Hz}$ and pulse width at $60 \mu \mathrm{s}$. Six versions of the algorithm were evaluated during the study to optimize the number of iterations and identify the most suitable target symptom to be used as feedback signal. Different algorithm versions were compared against each other by applying them in consecutive visits. After deciding on a most promising algorithm version, this version was used in all further study visits (subj. S0672G017 and subjects enrolled later, see Table 1). A subgroup of 10 subjects with this uniform algorithm version was analyzed separately.

To suggest new points (i.e., new stimulation settings) to explore in the two-dimensional space, the weighting method in the algorithm needs a minimum number of existing data points (see also starting point in Fig. 1E). To generate the number of data points required, a number of three to six (depending 
on the algorithm version) stimulation settings, scattered over the parameter-space, were chosen as predefined settings to be explored first at each algorithm-guided testing session.

Once the predefined settings (i.e., points on the two-dimensional spaces) are explored, the weighting method determines the next point to explore based on the score prediction map and distances between the explored points. First, the optimal vertical level is determined and afterwards, the algorithm explores radial steering on this vertical level. For every suggestion received, the DBS-settings were first evaluated for side-effects by the clinician. If the stimulation amplitude suggested by AgP led to side effects, the highest amplitude possible without side effects was chosen. After a wash-in time of at least 30 seconds, bradykinesia was assessed by a single motion-sensor task, and rigidity by a physician. Only the sensor-rated score was used as feedback for the algorithm.

Finally, the exploration method stops when predefined criteria for optimal setting are fulfilled. This is the case if the algorithm has exhausted the points in the two-dimensional space that could potentially lead to a score improvement, i.e., distance between explored and suggested points is below a predefined value.

\section{Statistics}

Full UPDRS-III and Kinesia One ${ }^{\mathrm{TM}}$ sensor scores were compared by median/interquartile range and programming time by mean/standard deviation. Wilcoxon signed-rank test was used to test for differences in UPDRS-III and sensor-based scores. Due to the exploratory nature of the study, no correction for multiple comparisons was performed. To explore potential factors that influence the effectiveness of AgP (measured as reduction of hemibody UPDRS-III by AgP compared to baseline/offstimulation-state) a mixed-effects (random intercept) model was used. Predictors tested in the mixedeffects model can be found in the supplementary methods. SPSS ${ }^{\circledast}$ was used for the mixed model, Matlab $^{\circledR}$ for all other analyses.

\section{Lead localization and active contact analysis}

Lead localizations were performed with the Lead-DBS toolbox (see also suppl. methods) [18]. Active contacts of AgP and SoC DBS-settings were compared with regards to their relative anatomical location to the STN, as well as to a literature-reported stimulation target [19]. The proximity of active contacts to this target coordinate has previously been shown to strongly correlate with clinical outcome [18], suggesting it could be an additional criterion for optimal contact selection. To validate this clinicalanatomical relationship in our dataset, distances between active contacts for both conditions (SoC and $\mathrm{AgP}$ ) and literature-reported stimulation target were first correlated with the relative changes in UPDRS-III hemibody scores, before comparing the distances between the conditions using a paired t- 
test. Of note, if multiple contacts were activated, a weighted mean distance was calculated according to the current distribution between the respective contacts. In order to determine whether activated contacts for each condition were closer to the literature-reported stimulation target than solely by chance, we applied a permutation approach [20] and calculated distances of randomly activated contacts $(10,000$ permutations for each condition) to the stimulation target. To retain the number of active contacts per lead and distribution of electrical current in the permuted data, active contacts were first permuted within each lead and then randomly assigned to different leads. Furthermore, permutations with non-adjacent active contact configurations were discarded, since they do not fulfill inclusion criteria of SoC as well as the restrictions of AgP settings. This way only the contact selection was randomized while other possible features, specific for each condition, were kept constant. Twosided t-tests were then performed between each permutation and the original sample in order to calculate median $p$-values for each condition. Finally, mean distances to the target were calculated across all permutations for each condition.

\section{Results}

In this study, 23 PD patients (4 female, mean age 61.9 years) who underwent STN-DBS were included (hereinafter referred to as "entire cohort"). One subject did not complete the study due to fatigue. In three subjects protocol deviations occurred (wrong programming of settings in one hemisphere $(n=2)$, reprogramming in one hemisphere due to side effects during blinded evaluation of AgP settings $(n=1))$. Therefore, these three subjects had to be excluded from the analyses using full UPDRS-III as the outcome parameter. In analyses using hemibody UPDRS-III as the outcome parameter, only the hemispheres $(n=3)$ affected by the protocol deviation were excluded. Two of the aforementioned subjects were in the subgroup with uniform algorithm version, thus 2 subjects ( 2 hemispheres) had to be excluded from analysis across subjects (hemispheres). Subjects' demographics and clinical data are shown in Table 1. SoC DBS-settings are shown in suppl. Table 1.

\section{Global clinical outcomes}

We found a statistically significant and clinically meaningful median improvement in full UPDRS-III scores for both, SoC (median 49\%, interquartile range $36-61 \%$ ) and $\mathrm{AgP}$ (37\%, interquartile range 18 - 54) DBS-settings compared to off-stimulation-state in the entire cohort (both $p<0.0002$ ). The median motion sensor score used for feedback was also significantly improved for SoC by $30 \%$ (interquartile range 16 - 45) and for AgP by 38\% (interquartile range 17 - 48) (both $p<0.003$ ). When considering the entire cohort, a significant difference between SoC and AgP in full UPDRS-III ( $n=19$, median 
(interquartile range) SoC: 21 (15.8 - 25.0), AgP: $25(20.8-33.5))$ and hemibody UPDRS-III $(n=41)$ scores was found ( $p=0.02$ in favor of SoC) but not for the sensor-based test battery ( $p=0.42$; Table 2 ).

In the analysis of the subgroup of subjects using only the latest and more advanced version of the algorithm, no significant differences were found between SoC and AgP for full UPDRS-III scores $(n=8$, median (interquartile range) SoC: 23 (14.0 - 27.5), AgP: 25 (18 - 34.5)) or sensor-based test battery. However, a significant difference in hemibody UPDRS-III scores $(n=18)$ remained between AgP and SoC $(p=0.05$ in favor of SoC, Table 2$)$.

Programming time for the AgP-loop (Fig. 1E) was $1.9 \pm 0.4$ hours and estimated programming time for SoC was $4.1 \pm 1.6$ hours in the entire cohort. Similar results were found for the subgroup (SoC: $4.0 \pm$ 1.4h, AgP $1.8 \pm 0.3 \mathrm{~h}$ ). Due to very different workflows in SoC and AgP, no significance testing was performed between the two programming modalities for time consumption. A mean number of 17.9 \pm 5.9 steps (i.e., algorithm cycles in Fig. 1, min. 7, max. 30 steps) per hemisphere was needed to reach the predefined stop criteria for AgP. As no reliable estimation of SoC-steps was possible, a comparison of steps needed for AgP and SoC as an exploratory endpoint was discarded. Side effects of AgP during blinded evaluation phase occurred in two subjects (delayed onset of dyskinesia and capsular stimulation). In three subjects the AgP-settings were chosen as Go-Home-Settings and one of those patients reported to have a sustained benefit from AgP-settings.

\section{Exploration of relevant factors for AgP}

A mixed-effects model for the complete dataset $(n=41$ hemispheres) showed four significant predictors for the effectiveness of AgP: a) range (difference between best and worst sensor-score obtained during the AgP-loop) of target-sensor-score (estimated regression coefficient $\beta=0.11 \pm 0.04$, $p=0.02) b)$ difference in stimulation amplitude between SoC and $\operatorname{AgP}(\beta=0.07 \pm 0.02, p=0.001) c+d)$ an interaction term containing hemibody UPDRS-III reduction by SoC and preoperative Levodoparesponse $(\beta=0.91 \pm 0.18, p<0.001)$. The relative model quality was considerably increased by backwards elimination and adding the interaction term (Akaike information criterion: all variables 7.6 / sensor-range and amplitude difference only 0.8 / with interaction term added -16.9). Amplitude difference and the interaction term remained significant predictors with regression coefficients in a similar range when the model was applied to the subgroup of subjects with uniform algorithm version (amplitude difference $\beta=0.06 \pm 0.02, p=0.009$; interaction term $\beta=0.84 \pm 0.25, p=0.007$ ). The range of the target sensor-score did not reach statistical significance in the subgroup but remained as a trend 
$(\beta=0.13 \pm 0.07, p=0.074)$. Stepwise increase of relative model quality was observed in the manner described for the full dataset (Akaike information criterion: 5.9 / 4.8 / -3.0).

\section{Lead localizations}

On average, $2.6 \pm 1.0$ and $2.8 \pm 1.4$ contacts were activated per lead in SoC and AgP DBS-settings, respectively (see also DBS-settings in suppl. table 1). No statistically significant differences were found with regard to stimulation amplitude (SoC: $3.0 \pm 1.1 \mathrm{~mA}$; AgP: $2.6 \pm 1.1 \mathrm{~mA}$ ). While $54 \%$ of all contacts were identically selected as active or inactive in both settings, some differences in contact selections were observed. Directional settings were selected by SoC in $36 \%$ of the cases, whereas AgP selected this kind of settings for $53 \%$ of the cases. From the contacts activated by SoC, $89 \%$ were located within or at the border of the STN, while for contacts activated by AgP, this percentage decreased to $70 \%$. Upper-level contacts were activated more often in AgP. This led to a difference in active contact locations compared to SoC $(p<0.001)$, with AgP active contacts being located on average $0.2 \mathrm{~mm}$ lateral, $0.4 \mathrm{~mm}$ anterior and $0.8 \mathrm{~mm}$ dorsal to SoC active contacts (Fig. 4). Dispersion of active contacts was smaller in SoC than in AgP (mean distance between active contacts $3.1 \pm 0.8 \mathrm{~mm}$ vs $3.9 \pm 1.1 \mathrm{~mm}$, $p<0.00001$ ). The distances between active contacts for all analyzed DBS-settings (i.e., SoC and AgP DBS-settings) and a literature-reported stimulation target were correlated to clinical improvements (Pearson $r=0.30, p<0.01$, Fig. 4). In SoC DBS-settings, active contacts were located closer to the target than randomly activated contacts (Permuted mean distance: $2.3 \pm 1.4 \mathrm{~mm}$, SoC: $1.8 \pm 1.0 \mathrm{~mm}$ with $\mathrm{p}<0.001)$. Active AgP contacts showed a trend towards being located closer to the stimulation target than contacts of permuted settings (Permuted mean distance: $2.3 \pm 1.4 \mathrm{~mm}$, AgP: $2.0 \pm 1.3 \mathrm{~mm}$ with $p=0.07)$. SoC active contacts were not located significantly closer to the stimulation target compared to AgP contacts $(p=0.11)$. Comparable results were obtained, when analyzing the subgroup of subjects with uniform algorithm version (see suppl. results).

\section{Discussion}

There is broad consensus that the complexity of DBS-programming with directional leads requires new approaches to successfully use the full range of settings available and reduce programming-burden for clinicians. Major strategies include feedback by wearable sensors, imaging-based selection of stimulation parameters, neurophysiological or even neurochemical monitoring $[2,5,21-24]$.

In our two-center study we investigated the new approach to replace the traditional monopolar review by a largely automated algorithm-guided testing strategy for stimulation settings with segmented 
leads in a randomized, crossover, double-blind setting. Both SoC and AgP DBS-settings resulted in clinically relevant improvement of motor symptoms compared to off-stimulation-state as measured by full UPDRS-III and motion sensor tasks. Considering the entire cohort, in whom different versions of the algorithm were tested, a statistically significant difference in UPDRS-III scores was found in favor of SoC. There was a difference of 4 points (12\%) in median UPDRS-III, which may just reach clinical relevance. Usually, a difference of 5 points or more is regarded as clinically relevant on a group level [25].

With the latest and most advanced version of the algorithm, outcomes did not differ significantly between SoC and AgP but could not be formally tested for non-inferiority. In this vein, the small sample size in this subgroup as well as a difference of 2 points (14\%) in median UPDRS-III in favor of SoC has to be considered and needs further evaluation in larger cohorts. Contacts activated in AgP DBS-settings showed a slightly larger average distance to the literature-reported stimulation target used in this work, and a greater spatial dispersion with a tendency to select upper contacts, compared to the contacts activated in SoC.

Previous studies using the same motion sensor system used in this study state a better test-retest reliability and sensitivity to changes in bradykinesia in DBS-patients compared to clinical rating $[26,27]$, which has been a rationale for using sensor measurements as main feedback for algorithmic programming. The model derived from our dataset suggests that AgP is mostly effective in patients, who show strong clinical treatment effects. One of the significant predictors for finding effective AgPsettings in terms of UPDRS-III improvement describes the effect magnitude of DBS on the sensormeasure used for feedback (larger range between best and worst score during AgP-loop leads to more effective AgP-settings). The other significant predictors describe the general treatment effect in a regular clinical setting (good effect of SoC-settings or Levodopa). This suggests that even though sensors are technically capable of capturing very small differences, in this study setting a quick and sufficiently strong clinical response is required to distinguish the effect of one specific DBS-setting from another setting or noise in behavioral data.

Furthermore, amplitudes were lower in AgP compared to SoC on average and the intraindividual stimulation amplitude difference between AgP and SoC partially explained the effectiveness of AgP. As active contact analysis showed AgP-contacts to be farther away from the stimulation target, one might hypothesize, that in AgP-contacts side effects might occur at lower amplitudes compared to SoCcontacts as AgP-contacts might be closer to adjacent anatomical structures causing side effects. This might hamper increasing the amplitude to an optimally effective level in AgP and lead to the observed effect of amplitude difference on AgP effectiveness. 
Up to now, there are only few studies investigating sensor-based DBS optimization. Recently, results from a cohort of patients with STN-DBS and linear 8-contact leads using the same algorithm as this study was published [28]. Similar to our study, SoC and AgP yielded significant improvements in UPDRSIII scores compared to off-stimulation-state and no significant differences were found between the two programming modalities. A significant reduction in steps needed to achieve the final DBS settings was also observed and in contrast to our study SoC programming was performed the same day as the study visit, thus allowing for a direct comparison. However, as only linear leads were investigated, the results only partially apply to our cohort. In directional leads both SoC and AgP most likely require more time and steps.

One study by Haddock et al. shows that AgP performed at least equally to SoC when only tremor rating is assessed [29]. In another study, Heldman et al. achieved a similar algorithm-guided improvement of $35.7 \%$ in tremor and bradykinesia but investigated a much smaller parameter-space due to conventional 4-ring-leads with only monopolar configurations [30]. In contrast, the parameter space of directional leads combined with multiple independent current sources in our study is far more complex (although in our study the algorithm only suggested cathodic stimulation settings on adjacent contacts). Furthermore, due to just unilateral testing in the study of Heldman et al., no comparison with standard of care settings or a control group was made [30]. In a similar study setting that used measurements of the same motion-sensor as feedback signal, AgP outperformed a clinical monopolar review in terms of DBS-effect on tremor and bradykinesia measured by the sensor system. However, the effect was mainly attributed to higher voltages chosen in AgP, and no blinded evaluation nor comparison in motor UPDRS scores was performed [31]. Importantly, in our cohort, stimulationamplitudes were non-significantly lower for AgP.

As PD symptoms show considerable interindividual differences, the signal used for feedback is a crucial factor for AgP. The clinically relevant DBS-effect may differ between patients (e.g., bradykinesia, tremor, gait disorder) and a sensor-based feedback system must be capable of measuring the symptom relevant to the individual patient or ideally, an individually weighted set of symptoms. One limitation of our study was that only a single motion-sensor measurement was used for feedback. Future designs should include a broader range of symptoms being measurable by sensors or physicians in order to continuously adapt stimulation specifically to a patient's individual symptom spectrum. Here, an advantage of sensor-based approaches, compared to imaging-guided strategies, is the potential to provide a continuous monitoring of symptoms and continuous adaption of PD therapy, including evaluation of different DBS-settings in a long-term outpatient context [32].

Several other approaches for guided programming, especially, image-guided approaches based on atlases $[20,33]$, tractography $[5,34]$ or resting-state MRI $[35,36]$, together with simulation of electrical 
fields or volume of activated tissue, can provide a high level of automation in the programming process. A recent study compared this method with traditional programming in PD patients with conventional 8-ring contact leads, three days after DBS-surgery. Based on unblinded patient judgment and blinded physician rating, results revealed that imaging-guided programming needed less programming time, but clinical effects were slightly inferior to traditional programming [37]. This is comparable to our results, where $\mathrm{AgP}$ and $\mathrm{SoC}$ were almost equal in terms of standardized ratings, but in most of the subjects, advantages of SoC were noticeable for the blinded physician (e.g., global impression in gait assessment, small but visible differences in bradykinesia), that did not lead to a change in the respective UPDRS-III item according to rating criteria. This clinical observation is supported by results of our imaging data, which shows a difference in location of the activated contacts for AgP and SoC with regards to the z-axis on the lead, a smaller dispersion of active contacts for SoC, and a higher portion of contacts inside the STN for SoC. The combination of imaging-guided approaches and sensor-feedback-based adjustment could improve AgP strategies in the future.

Several limitations need to be considered when interpreting the results of our study. Because of the exploratory nature of the study, the algorithm was adapted several times, so the subgroup of subjects with a uniform version of the algorithm is small, which did not allow non-inferiority testing. Another limitation is the feedback to the algorithm using only a single sensor-based parameter. This might account for the fact, that while the sensor-based parameter was efficiently optimized by the algorithm, advantages for SoC were noticeable for subjects and the blinded physician. Using multiple symptoms for sensor-based feedback and reducing the parameter space based on imaging could improve AgP approaches in the future. Finally, SoC time consumption was solely based on the physician's estimation and medical history. Therefore, it is not possible to make a valid, direct comparison between SoC and AgP in terms of time consumption. However, considering clinical experience the usual programming time in clinical routine exceeds $2 \mathrm{~h}$ per patient and our results support that future development of effective AgP may help to reduce the programming burden for clinicians.

In summary, we provide evidence for feasibility of algorithm-guided programming in a prospective, randomized, double-blind trial applying motion-sensor-guided programming in directional leads. Although not providing evidence for non-inferiority of algorithm-guidance in long-term clinical application, our results may guide future development in the field of algorithm-guided programming. 


\section{Suppl. Methods:}

\section{Mixed-effects model}

Potential predictors were 1) reduction of hemibody UPDRS-III by SoC settings in blinded evaluation phase, 2,3 ) reduction of sensor-based test battery hemibody scores by SoC settings and AgP in blinded evaluation-phase, 4) range (maximum-minimum, excluding outliers, i.e., 1.5 interquartile range above third quartile) of target sensor-based score (used for algorithm feedback during AgP), $5,6,7)$ physician-assessed rigidity and sensor-based subitems speed and amplitude for final AgP settings compared to median of other settings tested during AgP iterations (difference between results for final settings and median), 8) algorithm software version 9) number of iterations to determine AgP settings, 10) minimum distance between lead and a literature-reported stimulation target[19], 11) UPDRS-III reduction in preoperative L-Dopa challenge, 12) Stimulation amplitude differences between SoC and AgP. Variables were then subsequently selected by backwards elimination (repeated exclusion of variable with highest non-significant $p$-value). Interaction terms for clinically reasonable interactions in the model were tested. Improvement of relative model quality during variable selection was checked by Akaike information criterion.

\section{Lead localization}

All leads were localized in Montreal Neurological Institute (MNI) space (2009b, nonlinear, asymmetric) according to the pipeline implemented in Lead-DBS Matlab ${ }^{\text {TM }}$ toolbox[18]. In one of the study centers (Charité) lead localizations are routinely performed after DBS-implant and occasionally used as a programming aid. Rotation of directional leads was determined by the "DiODe" algorithm[38,39]. Lefthemispheric leads were flipped nonlinearly to the contralateral side. All steps were visually inspected and manually corrected, if necessary, to ensure data quality. The stimulation target has been previously defined in a meta-analysis[19] and transformed to Montreal Neurological Institute space in probabilistic fashion[40].

\section{Suppl. Results:}

Similar results to the entire cohort were obtained, when analyzing the subset of subjects with uniform algorithm version:

On average $2.7 \pm 1.3$ and $3.3 \pm 1.6$ contacts were activated per lead in SoC and AgP settings, respectively. No statistically significant differences were found with regard to stimulation amplitude (SoC: $3.3 \pm 1.2 \mathrm{~mA}$; AgP: $2.7 \pm 1.3 \mathrm{~mA}$ ). While $43 \%$ of all contacts were identically selected as active or inactive in both settings, some differences in contact selections were observed. In SoC directional 
settings were selected in $44 \%$ of cases opposed to $28 \%$ in AgP. Contacts were located within or at the border of STN in $93 \%$ of active SoC contacts but only in $72 \%$ for AgP settings. Upper contact levels were selected more often in AgP. This led to a difference in active contact locations compared to SoC $(p=0.047)$ with average AgP contacts being located $0.2 \mathrm{~mm}$ lateral, $0.4 \mathrm{~mm}$ anterior and $0.6 \mathrm{~mm}$ dorsal to SoC contacts (Fig. 4). The dispersion of active contacts was smaller in SoC than in AgP (mean distance between active contacts $3.1 \mathrm{~mm} \pm 0.9$ vs $4.0 \mathrm{~mm} \pm 1.4, \mathrm{p}<0.01$ ). The distances between active contacts and a literature-derived stimulation target[19,40] were correlated to clinical improvements (Pearson $r=0.34, p=0.044$, Fig. 4). In SoC settings active contacts were located closer to the target than randomly selected contacts (Permuted mean distance: $2.3 \pm 1.4 \mathrm{~mm}$, SoC: $1.8 \pm 1.2 \mathrm{~mm}$ with $p=0.02$ ). Active contacts in AgP settings were not located closer to the stimulation target (Permuted mean distance: $2.3 \pm 1.4 \mathrm{~mm}, \mathrm{AgP}: 2.2 \pm 1.7 \mathrm{~mm}$ with $\mathrm{p}=0.60$ ). A trend towards SoC contacts being located closer to the stimulation target compared to AgP contacts was found $(p=0.14)$. 


\section{References}

[1] Limousin P, Krack P, Pollak P, Benazzouz A, Ardouin C, Hoffmann D, Benabid A (1998) Electrical stimulation of the subthalamic nucleus in advanced Parkinson's disease. The New England journal of medicine 339, 1105-1111.

[2] Krack P, Volkmann J, Tinkhauser G, Deuschl G (2019) Deep Brain Stimulation in Movement Disorders: From Experimental Surgery to Evidence-Based Therapy. Movement Disorders 34, 1795-1810.

[3] Volkmann J, Albanese A, Antonini A, Chaudhuri KR, Clarke CE, de Bie RMA, Deuschl G, Eggert K, Houeto J-L, Kulisevsky J, Nyholm D, Odin P, Østergaard K, Poewe W, Pollak P, Rabey JM, Rascol O, Ruzicka E, Samuel M, Speelman H, Sydow O, Valldeoriola F, van der Linden C, Oertel W (2013) Selecting deep brain stimulation or infusion therapies in advanced Parkinson's disease: an evidence-based review. J Neurol 260, 2701-2714.

[4] Kleiner-Fisman G, Herzog J, Fisman DN, Tamma F, Lyons KE, Pahwa R, Lang AE, Deuschl G (2006) Subthalamic nucleus deep brain stimulation: summary and meta-analysis of outcomes. Mov Disord 21 Suppl 14, S290-304.

[5] Horn A (2019) The impact of modern-day neuroimaging on the field of deep brain stimulation. Current Opinion in Neurology 32, 511-520.

[6] Steigerwald F, Matthies C, Volkmann J (2019) Directional Deep Brain Stimulation. Neurotherapeutics 16, 100-104.

[7] Dembek TA, Reker P, Visser-Vandewalle V, Wirths J, Treuer H, Klehr M, Roediger J, Dafsari HS, Barbe MT, Timmermann L (2017) Directional DBS increases side-effect thresholds-A prospective, double-blind trial. Movement disorders : official journal of the Movement Disorder Society 32, 1380-1388.

[8] Nguyen TAK, Nowacki A, Debove I, Petermann K, Tinkhauser G, Wiest R, Schüpbach M, Krack P, Pollo C (2019) Directional stimulation of subthalamic nucleus sweet spot predicts clinical efficacy: Proof of concept. Brain Stimulation 12, 1127-1134.

[9] Barbe MT, Dembek TA, Becker J, Raethjen J, Hartinger M, Meister IG, Runge M, Maarouf M, Fink GR, Timmermann $L$ (2014) Individualized current-shaping reduces DBS-induced dysarthria in patients with essential tremor. Neurology 82, 614-619.

[10] Reker P, Dembek TA, Becker J, Visser-Vandewalle V, Timmermann L (2016) Directional deep brain stimulation: A case of avoiding dysarthria with bipolar directional current steering. Parkinsonism \& related disorders $31,156-158$.

[11] Gunduz A, Foote KD, Okun MS (2017) Reengineering deep brain stimulation for movement disorders: Emerging technologies. Curr Opin Biomed Eng 4, 97-105.

[12] Picillo M, Lozano AM, Kou N, Puppi Munhoz R, Fasano A (2016) Programming Deep Brain Stimulation for Parkinson's Disease: The Toronto Western Hospital Algorithms. Brain Stimulation 9, 425-437.

[13] Jens Volkmann, Elena Moro, Rajesh Pahwa (2006) Basic algorithms for the programming of deep brain stimulation in Parkinson's disease. Movement Disorders 21,.

[14] Wagle Shukla A, Zeilman P, Fernandez H, Bajwa JA, Mehanna R (2017) DBS Programming: An Evolving Approach for Patients with Parkinson's Disease. Parkinsons Dis 2017, 8492619.

[15] Lieber B, Taylor BES, Appelboom G, McKhann G, Connolly ES (2015) Motion Sensors to Assess and Monitor Medical and Surgical Management of Parkinson Disease. World Neurosurg 84, 561-566.

[16] Mitchell KT, Starr PA (2020) Smart neuromodulation in movement disorders. In Handbook of Clinical Neurology Elsevier, pp. 153-161.

[17] Giuffrida JP, Riley DE, Maddux BN, Heldman DA (2009) Clinically deployable Kinesia technology for automated tremor assessment. Mov Disord 24, 723-730.

[18] Horn A, Li N, Dembek TA, Kappel A, Boulay C, Ewert S, Tietze A, Husch A, Perera T, Neumann W-JJ, Reisert M, Si H, Oostenveld R, Rorden C, Yeh F-CC, Fang Q, Herrington TM, Vorwerk J, Kühn AA (2019) Lead-DBS v2: Towards a comprehensive pipeline for deep brain stimulation imaging. Neurolmage 184, 293-316.

[19] Caire F, Ranoux D, Guehl D, Burbaud P, Cuny E (2013) A systematic review of studies on anatomical position of electrode contacts used for chronic subthalamic stimulation in Parkinson's disease. Acta Neurochir 155, 1647-1654.

[20] Dembek TA, Roediger J, Horn A, Reker P, Oehrn C, Dafsari HS, Li N, Kühn AA, Fink GR, Visser-Vandewalle V, Barbe MT, Timmermann L (2019) Probabilistic sweet spots predict motor outcome for deep brain stimulation in Parkinson disease. Annals of Neurology 86, 527-538. 
[21] Cagnan H, Denison T, Mclntyre C, Brown P (2019) Emerging technologies for improved deep brain stimulation. Nat Biotechnol 37, 1024-1033.

[22] Hell F, Köglsperger T, Mehrkens J, Boetzel K (2018) Improving the Standard for Deep Brain Stimulation Therapy: Target Structures and Feedback Signals for Adaptive Stimulation. Current Perspectives and Future Directions. Cureus 10, e2468.

[23] Anderson D, Beecher G, Ba F (2017) Deep Brain Stimulation in Parkinson's Disease: New and Emerging Targets for Refractory Motor and Nonmotor Symptoms. Parkinson's Disease 2017, 1-13.

[24] Kühn AA, Volkmann J (2017) Innovations in deep brain stimulation methodology. Movement disorders : official journal of the Movement Disorder Society 32, 11-19.

[25] Schrag A, Sampaio C, Counsell N, Poewe W (2006) Minimal clinically important change on the unified Parkinson's disease rating scale. Mov Disord 21, 1200-1207.

[26] Heldman DA, Espay AJ, LeWitt PA, Giuffrida JP (2014) Clinician versus machine: reliability and responsiveness of motor endpoints in Parkinson's disease. Parkinsonism Relat Disord 20, 590-595.

[27] Heldman DA, Urrea-Mendoza E, Lovera LC, Schmerler DA, Garcia X, Mohammad ME, McFarlane MCU, Giuffrida JP, Espay AJ, Fernandez HH (2017) App-Based Bradykinesia Tasks for Clinic and Home Assessment in Parkinson's Disease: Reliability and Responsiveness. J Parkinsons Dis 7, 741-747.

[28] Sasaki F, Oyama G, Sekimoto S, Nuermaimaiti M, Iwamuro H, Shimo Y, Umemura A, Hattori N (2021) Closed-loop programming using external responses for deep brain stimulation in Parkinson's disease. Parkinsonism Relat Disord 84, 47-51.

[29] Haddock A, Mitchell KT, Miller A, Ostrem JL, Chizeck HJ, Miocinovic S (2018) Automated Deep Brain Stimulation Programming for Tremor. IEEE Trans Neural Syst Rehabil Eng 26, 1618-1625.

[30] Heldman DA, Pulliam CL, Urrea Mendoza E, Gartner M, Giuffrida JP, Montgomery EB, Espay AJ, Revilla FJ (2016) Computer-Guided Deep Brain Stimulation Programming for Parkinson's Disease. Neuromodulation 19, 127-132.

[31] Pulliam CL, Heldman DA, Orcutt TH, Mera TO, Giuffrida JP, Vitek JL (2015) Motion sensor strategies for automated optimization of deep brain stimulation in Parkinson's disease. Parkinsonism \& related disorders 21, 378-382.

[32] Mera TO, Burack MA, Giuffrida JP (2013) Objective Motion Sensor Assessment Highly Correlated with Scores of Global Levodopa-Induced Dyskinesia in Parkinson's Disease. Journal of Parkinson's Disease 3, 399-407.

[33] Ewert S, Plettig P, Li N, Chakravarty MM, Collins DL, Herrington TM, Kühn AA, Horn A (2018) Toward defining deep brain stimulation targets in MNI space: A subcortical atlas based on multimodal MRI, histology and structural connectivity. Neurolmage 170, 271-282.

[34] Krishna V, Sammartino F, Rabbani Q, Changizi B, Agrawal P, Deogaonkar M, Knopp M, Young N, Rezai A (2019) Connectivity-based selection of optimal deep brain stimulation contacts: A feasibility study. Ann Clin Transl Neurol 6, 1142-1150.

[35] Horn A, Wenzel G, Irmen F, Huebl J, Li N, Neumann W-J, Krause P, Bohner G, Scheel M, Kühn AA (2019) Deep brain stimulation induced normalization of the human functional connectome in Parkinson's disease. Brain 142, 3129-3143.

[36] Horn A, Reich M, Vorwerk J, Li N, Wenzel G, Fang Q, Schmitz-Hübsch T, Nickl R, Kupsch A, Volkmann J, Kühn AA, Fox MD (2017) Connectivity Predicts deep brain stimulation outcome in Parkinson disease. Annals of neurology 82, 67-78.

[37] Pavese N, Tai YF, Yousif N, Nandi D, Bain PG (2019) Traditional Trial and Error versus Neuroanatomic 3Dimensional Image Software-Assisted Deep Brain Stimulation Programming in Patients with Parkinson Disease. World Neurosurg.

[38] Sitz A, Hoevels M, Hellerbach A, Gierich A, Luyken K, Dembek TA, Klehr M, Wirths J, Visser-Vandewalle $V$, Treuer $H$ (2017) Determining the orientation angle of directional leads for deep brain stimulation using computed tomography and digital x-ray imaging: A phantom study. Medical Physics 44, 44634473.

[39] Hellerbach A, Dembek TA, Hoevels M, Holz JA, Gierich A, Luyken K, Barbe MT, Wirths J, VisserVandewalle V, Treuer H (2018) DiODe: Directional Orientation Detection of Segmented Deep Brain Stimulation Leads: A Sequential Algorithm Based on CT Imaging. SFN 96, 335-341.

[40] Horn A, Kühn AA, Merkl A, Shih L, Alterman R, Fox M (2017) Probabilistic conversion of neurosurgical DBS electrode coordinates into MNI space. Neuroimage 150, 395-404. 
Figure 1

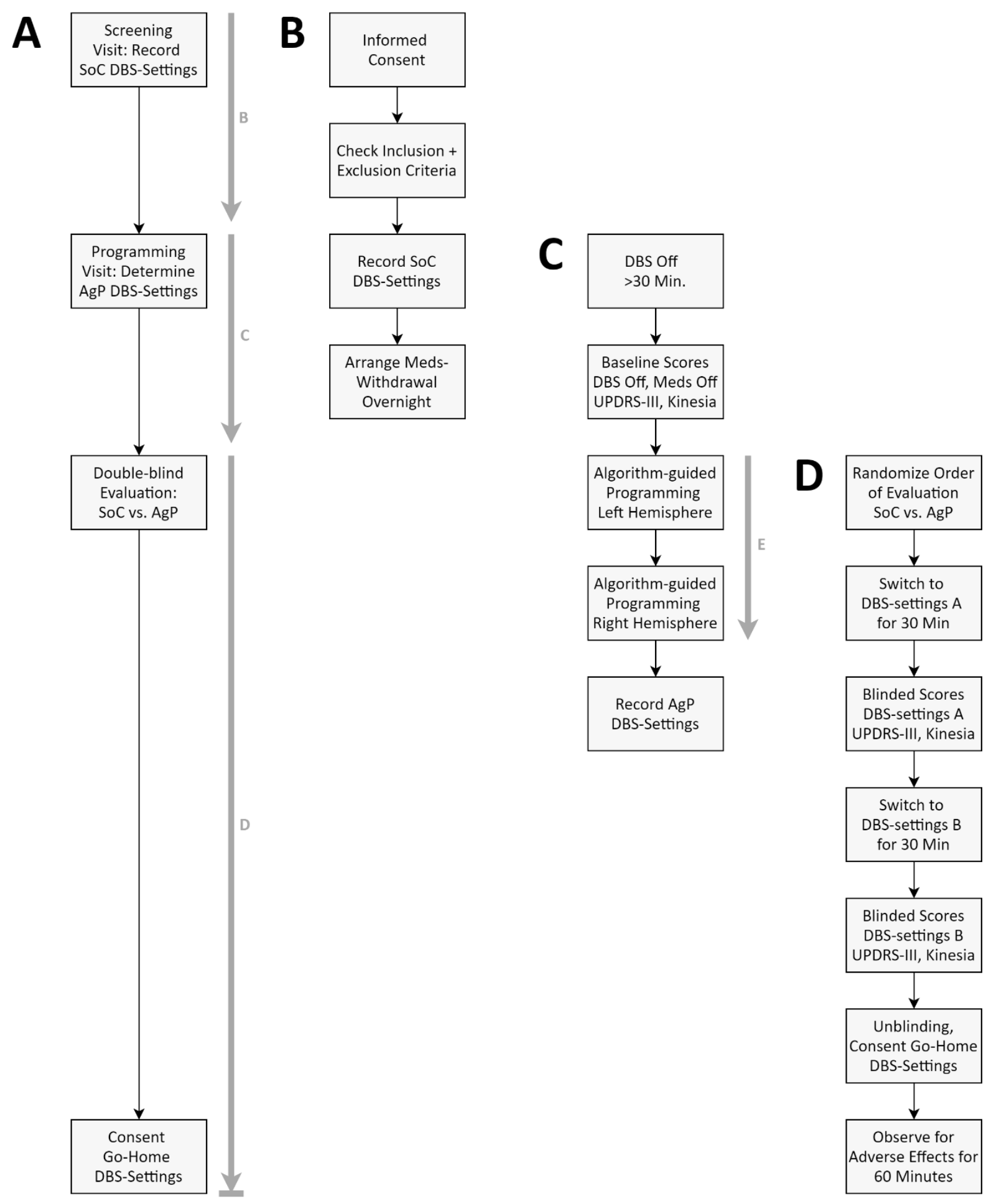




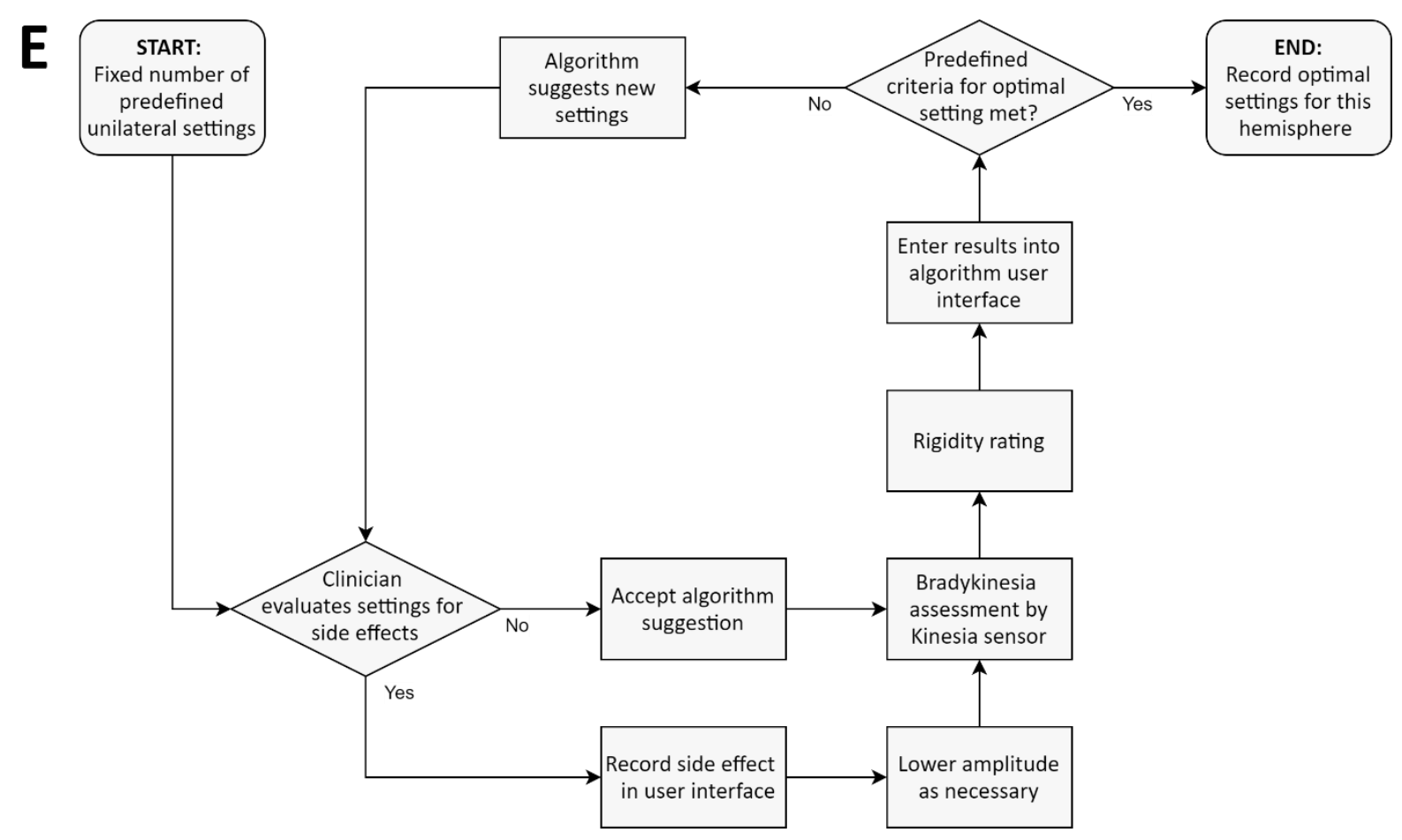

Fig. 1. Visual representation of the study design and the algorithm-guided programming-loop. A: Overview of the study workflow. The bold, grey arrows indicate the panels showing a detailed description of this section of the study protocol. B: Screening Visit. After informed consent, SoC DBSsettings were recorded. SoC DBS-settings are defined as the settings established by clinical routine prior to enrollment in the study. Subjects were instructed for an overnight withdrawal of dopaminergic medication. C: Programming visit. After switching off DBS for at least 30 minutes, baseline UPDRS-III and Kinesia sensor test battery were performed. Then AgP DBS-settings were obtained by an iterative sensor-feedback-loop (panel E) for left hemisphere first, and then for right hemisphere. D: Subjects were then randomized into one of two study arms that differed in the order by which settings (SoC vs. $\mathrm{AgP}$ ) were being tested. "DBS-settings $\mathrm{A}$ " here refers to the settings tested first and "DBS-settings B" to the ones tested second. After double-blind evaluation, physician and subject agreed on go-homesettings, which could be either SoC or AgP. E: Illustration of the AgP-loop. The exact position of the AgP-loop in the whole study workflow is shown by the arrow in panel C. For a detailed description of the AgP loop see "Methods"-section. 

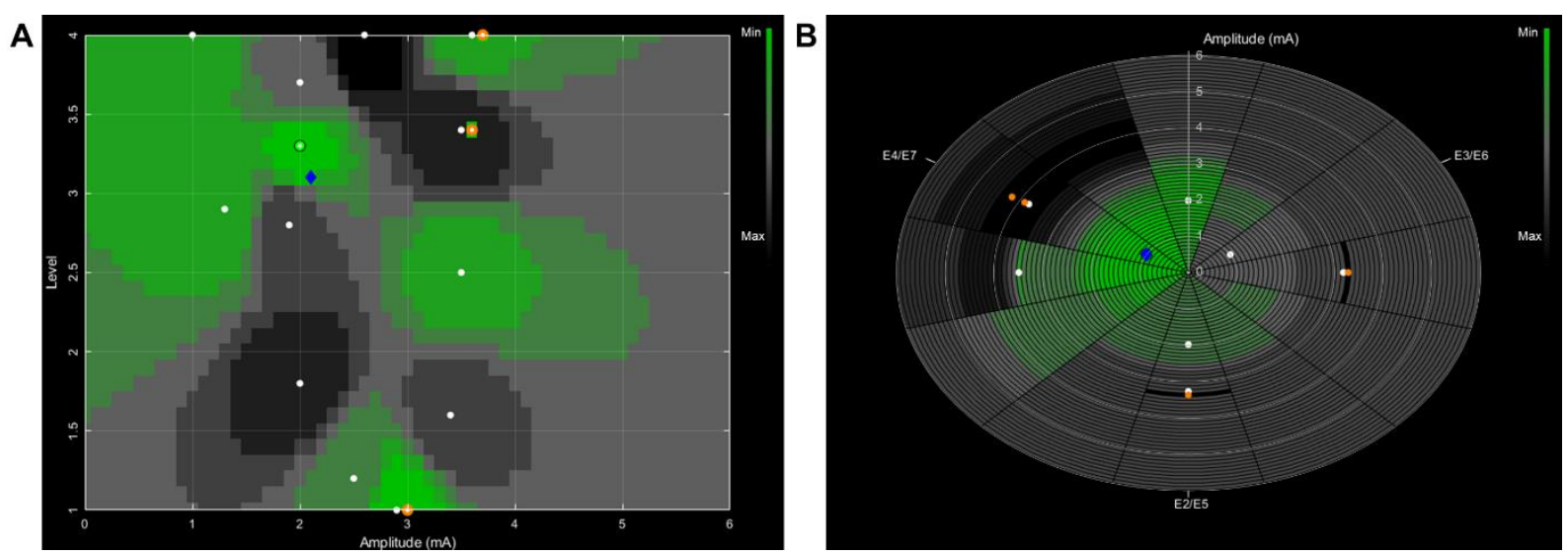

Fig. 2. Two-dimensional representation of clinical DBS effects of different stimulation settings in a single subject. Each stimulation setting consists of a contact configuration and a stimulation amplitude. A: Cartesian representation of clinical effects for ring mode stimulation settings. The $y$-axis corresponds to the four contact levels of the Boston Scientific directional lead. The $x$-axis corresponds to the stimulation amplitude. B: Polar representation of clinical effects for directional mode stimulation settings. Each of the slices on the plot corresponds to a $30^{\circ}$ angle. The labels (e.g. E2/5) indicate the radial orientation of the tripartite contacts in Boston Scientific directional leads, each spanning $120^{\circ}$. The radius of the slices corresponds to the stimulation amplitude.

For both representations, each plotted point (white circles) represents the motion sensor score of a previously suggested and tested stimulation. The color-coding of the areas in between the tested stimulation settings (white circles) represents the score predicted by the algorithm. Green colors represent a lower (favorable) value of the predicted score, whereas darker colors represent a higher value.

The blue diamond represents the current suggestion of the algorithm for the next stimulation setting to be tested. In panel $A$ this blue diamond refers to a ring mode stimulation setting with a total amplitude of $2.1 \mathrm{~mA}$ (upper tripartite contacts, labeled 5, 6 and 7 with $30 \%$ and uppermost ring contact, labeled 8 with $10 \%$ of total current). In panel $B$ the blue diamond refers to a directional stimulation setting with a total amplitude of $1 \mathrm{~mA}$. The radial current steering is directed to the right-posterior segments of the tripartite contacts (contact 7 with $70 \%$ and contact 8 with $30 \%$ of total current). 


\section{Figure 3}

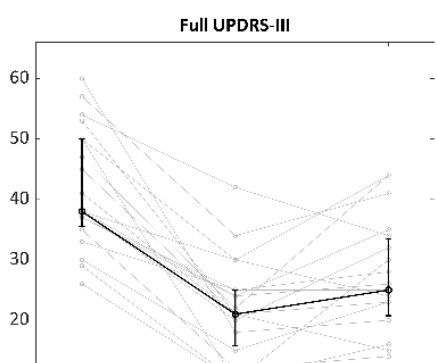

A
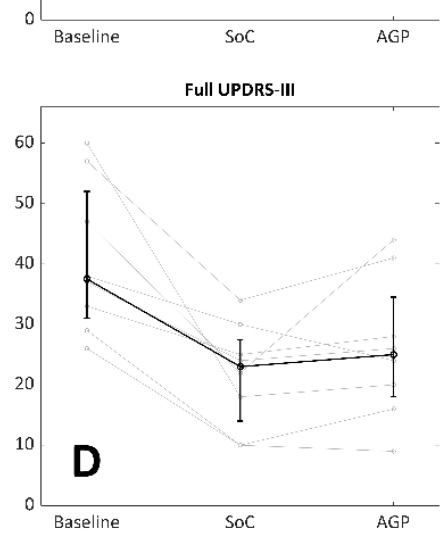
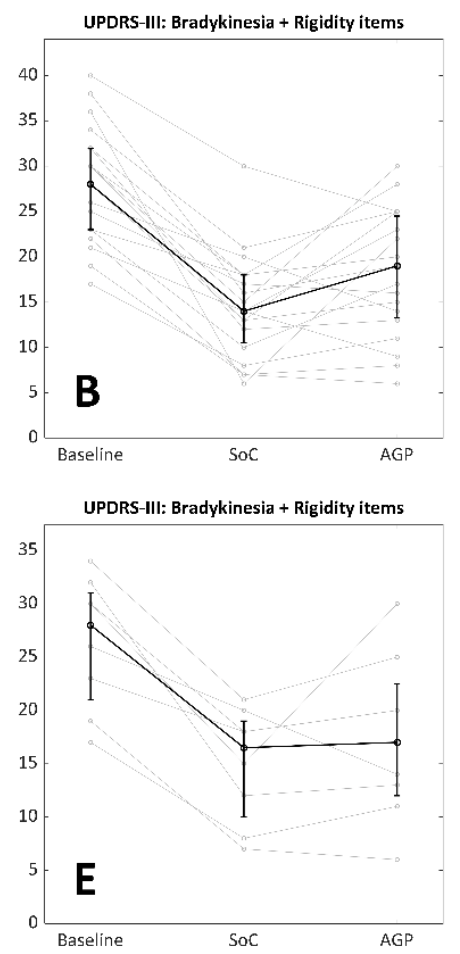
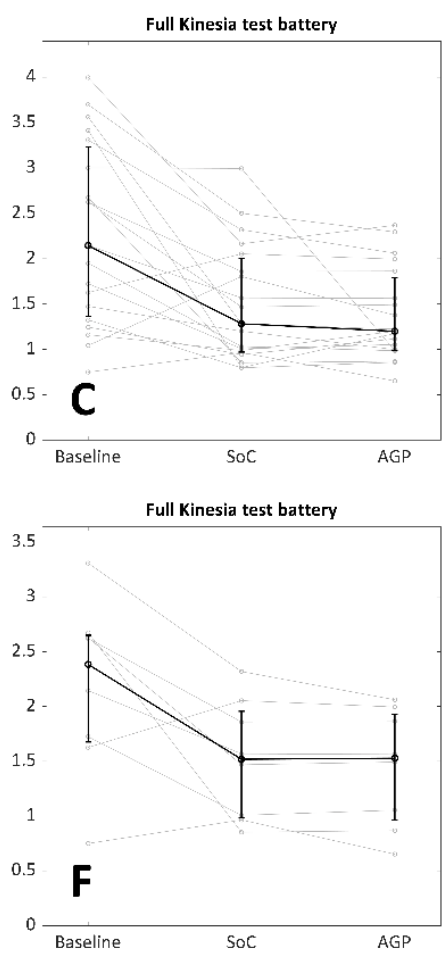

Fig. 3. Outcome measures of double-blind evaluation (SoC vs. AgP) in randomized order. The bold black line shows median values with interquartile range. A: Full UPDRS-III scores, entire cohort. B: UPDRSIII-items related to bradykinesia and rigidity, entire cohort. Note the different scaling of the $y$-axis compared to panel A. C: Median score of motion-sensor full test battery (rest tremor, finger taps, hand grasps, all bilateral), entire cohort. Symptom severity in every task is rated by a proprietary algorithm of the manufacturer on a scale from 0 (best) to 4 (worst) D-F: same data for subjects in the subgroup with uniform algorithm version. 


\section{Figure 4}

A

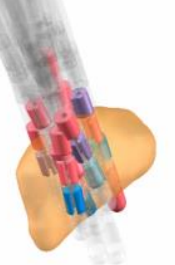

C

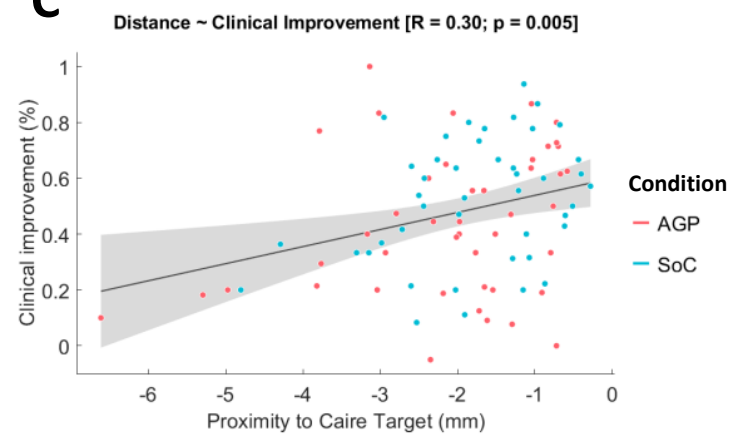

B
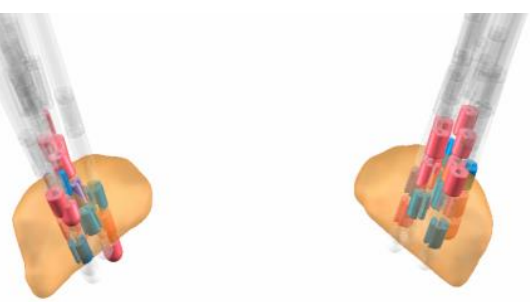

D

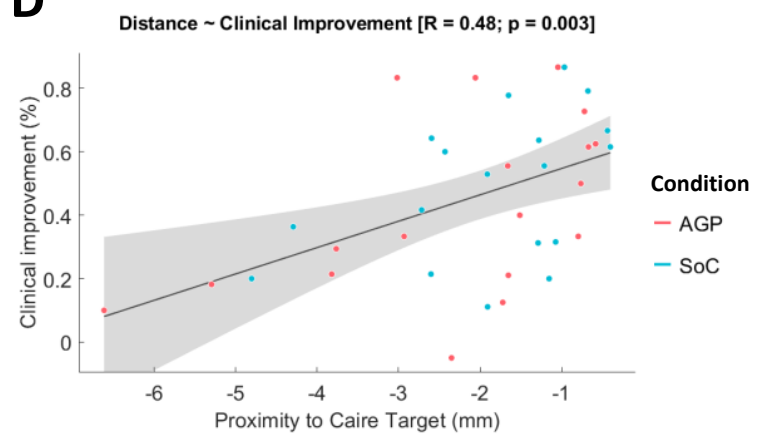

Fig. 4. A-B: Reconstructed lead localizations in the STN (orange) by Lead-DBS Matlab toolbox of the full study cohort (left) or the subgroup with uniform algorithm version (right). Red: AgP-contacts, blue: SoC-contacts, purple: shared contacts. Note the dorsally located clustering of red (AgP) contacts. C-D: Correlation plots for the full cohort (left) and subgroup (right) between distance of active contact to a literature reported stimulation target and clinical improvement (hemibody UPDRS-III score, percent improvement compared to baseline, i.e., off-medication-state, off-stimulation-state). 


\section{Table 1}

\begin{tabular}{|c|c|c|c|c|c|c|c|c|}
\hline & & & & Demog & aphics & & & \\
\hline & Age & Gender & $\begin{array}{c}\text { Age at disease } \\
\text { onset }\end{array}$ & $\begin{array}{c}\text { Disease } \\
\text { duration at } \\
\text { surgery (years) }\end{array}$ & $\begin{array}{l}\text { Months since } \\
\text { surgery }\end{array}$ & $\begin{array}{l}\text { UPDRS Meds } \\
\text { Off pre surgery }\end{array}$ & $\begin{array}{l}\text { Improvement } \\
\text { Percentage } \\
\text { Levodopa } \\
\text { challenge }\end{array}$ & $\begin{array}{l}\text { Improvement } \\
\text { Percentage SoC- } \\
\text { DBS (Meds Off } \\
\text { state) }\end{array}$ \\
\hline S0672G001 & 58 & Male & 37 & 18 & 33 & 39 & 69,2 & 32,4 \\
\hline S0672G002 & 51 & Male & 42 & 5 & 43 & 37 & 37,8 & 60,4 \\
\hline S0672G003 ${ }^{b}$ & 54 & Male & 38 & 14 & 20 & 27 & 85,2 & 52,2 \\
\hline S0672G004 & 74 & Male & 64 & 9 & 12 & 40 & 42,5 & 40 \\
\hline S0672G005 & 49 & Male & 41 & 8 & 7 & 69 & 49,3 & 80 \\
\hline S0672G006 & 60 & Male & 52 & 8 & 7 & 29 & 79,3 & 50 \\
\hline S0672G007 & 55 & Male & 43 & 11 & 6 & 44 & 61,4 & 65,7 \\
\hline S0672G008 & 64 & Male & 50 & 13 & 8 & 52 & 57,7 & 48,8 \\
\hline S0672G010 & 63 & Male & 46 & 16 & 10 & 61 & 60,7 & 47,4 \\
\hline $\mathrm{S} 0672 \mathrm{G} 011^{\mathrm{a}, \mathrm{b}}$ & 70 & Female & 51 & 18 & 6 & 35 & 57,1 & 58,1 \\
\hline $\mathrm{S} 0672 \mathrm{G} 012^{\mathrm{a}}$ & 62 & Male & 50 & 11 & 12 & 49 & 59,2 & 21,1 \\
\hline S0672G013 & 66 & Male & 55 & 10 & 6 & 78 & 39,7 & 22,2 \\
\hline $\mathrm{S} 0672 \mathrm{G} 014^{\mathrm{a}}$ & 71 & Male & 59 & 11 & 12 & 32 & 50 & 24,2 \\
\hline S0672G015 & 63 & Male & 49 & 14 & 6 & 53 & 66 & 53,3 \\
\hline S0672G016 & 68 & Female & 50 & 18 & 9 & 59 & 22 & 36,8 \\
\hline $\mathrm{S} 0672 \mathrm{G} 017^{\mathrm{a}}$ & 67 & Male & 42 & 23 & 15 & 64 & 43,8 & 40,4 \\
\hline $\mathrm{S} 0672 \mathrm{G} 018^{\mathrm{a}}$ & 65 & Male & 56 & 8 & 8 & 48 & 54,2 & 65,5 \\
\hline $\mathrm{S} 0672 \mathrm{G} 019^{\mathrm{a}}$ & 57 & Male & 38 & 18 & 6 & 37 & 37,8 & 61,5 \\
\hline $\mathrm{S} 0672 \mathrm{G} 020^{\mathrm{a}}$ & 59 & Female & 45 & 13 & 7 & 60 & 81,7 & 70 \\
\hline S0309G002 & 63 & Male & 46 & 17 & 7 & 25 & 56 & 35,9 \\
\hline $\mathrm{S} 0309 \mathrm{G}_{00} \mathrm{3}^{\mathrm{a}}$ & 63 & Male & 48 & 15 & 8 & 40 & 55 & 53,2 \\
\hline $\mathrm{S}_{0309 G 004^{\mathrm{a}}}$ & 59 & Female & 46 & 11 & 17 & 36 & 58,3 & 35,1 \\
\hline mean full & & & & & & & & \\
\hline $\begin{array}{l}\text { cohort } \\
n=22\end{array}$ & 61,9 & 4 female & 47,6 & 13,1 & 12,0 & 42,0 & 56,6 & 49,4 \\
\hline $\begin{array}{l}\text { subgroup } \\
n=10\end{array}$ & 63,6 & 3 female & 48,1 & 14,5 & 9,8 & 38,5 & 55,5 & 46,8 \\
\hline
\end{tabular}

Table 1: Demographic data of study subjects. The column "Improvement Levodopa challenge" relates to preoperative Levodopa challenge for DBSevaluation. a) Subgroup with uniform algorithm version, b) subjects with one hemisphere excluded from hemisphere-wise analysis. Subject S0672G009 did not complete the study due to fatigue and was excluded from any analysis. 


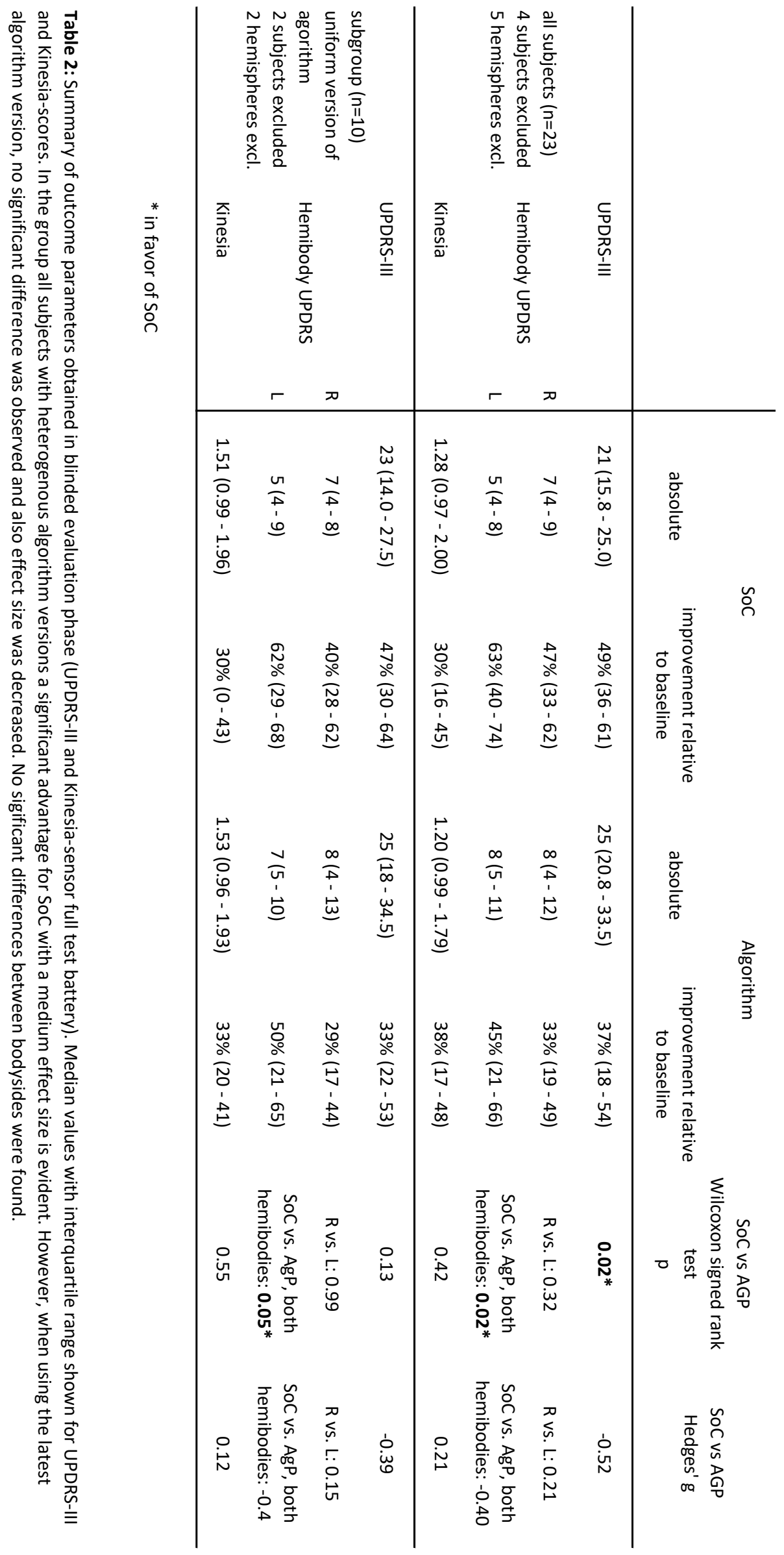




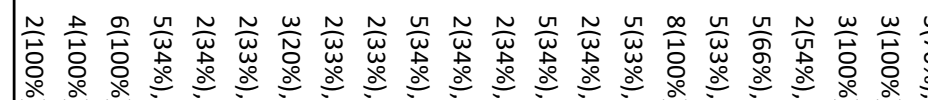

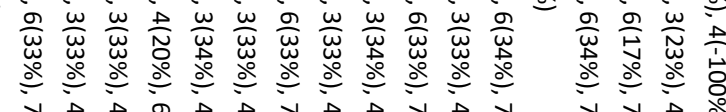

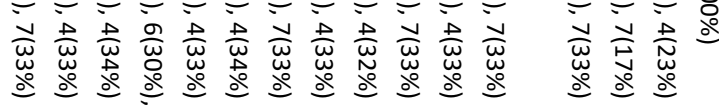

啇

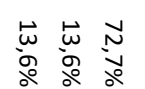

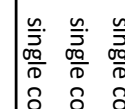

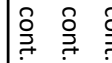

$\underset{\infty}{\infty}$

$\underset{\sim}{\stackrel{n}{N}}$

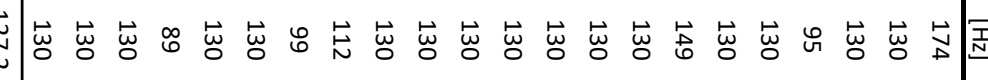

ज

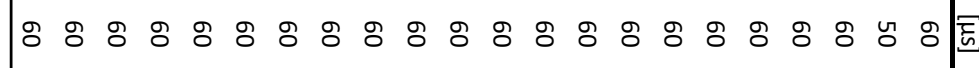

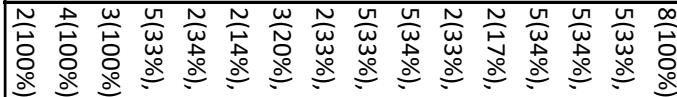

$\frac{\sigma}{\omega} \frac{\omega}{\omega} \frac{\omega}{\omega} \frac{D}{N} \frac{\omega}{\omega} \frac{\sigma}{\omega} \frac{O}{\omega} \frac{\omega}{\omega} \frac{\omega}{\sigma} \frac{O}{\omega} \frac{O}{\omega} \frac{O}{\omega}$

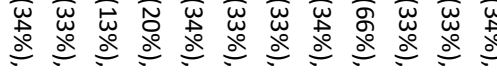

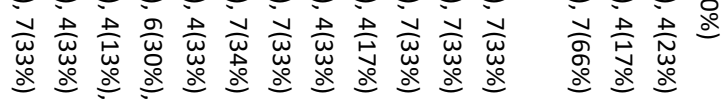

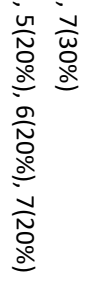

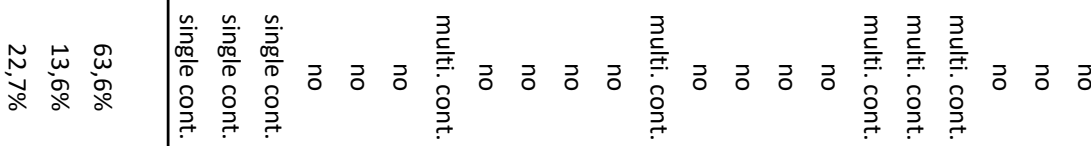

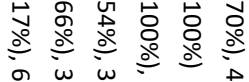

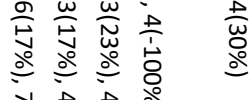

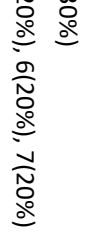

$\stackrel{\omega}{\sim}$

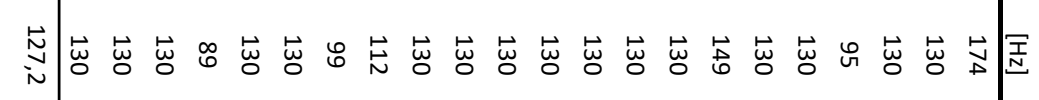

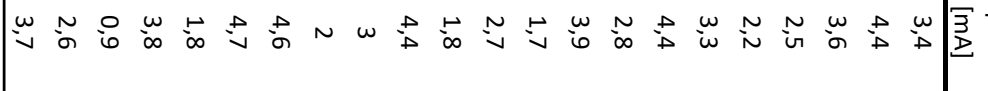

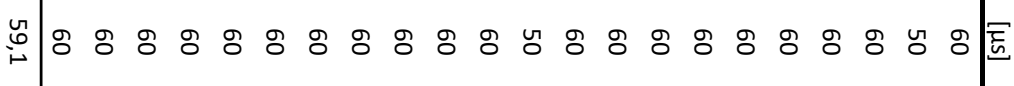




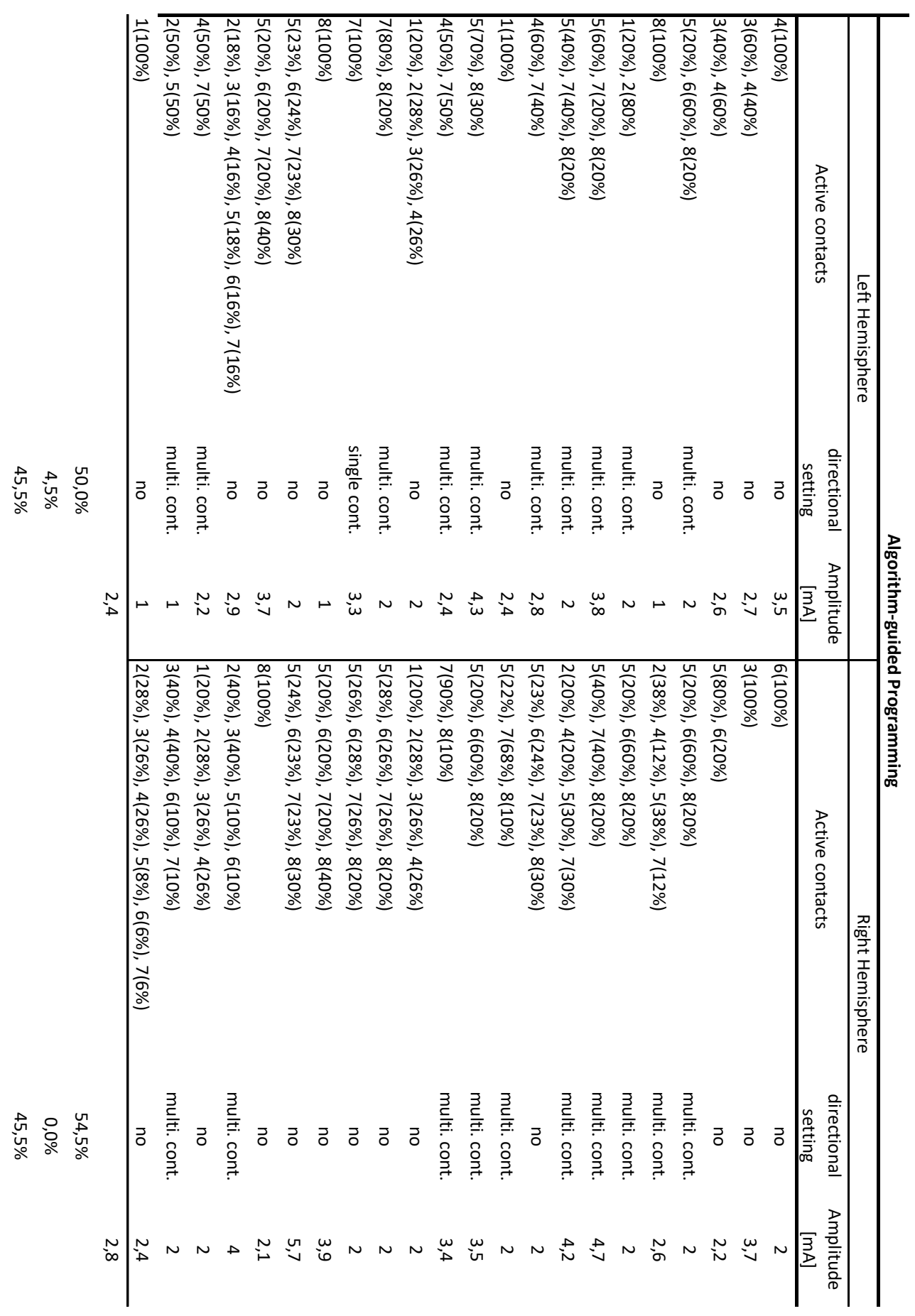




\section{Declaration of Interest}

GRW received travel expenses and attendance fees by Boston Scientific and Ipsen Pharma.

JR has nothing to disclose.

EG declares travel and attendance grants from Abbvie, Abbott, Medtronic and Bial.

HS, LJP and KW are full-time eployees of Boston Scientific.

MPN received Boston scientific study fees and Abbott study fees.

AAK declares that she is on the advisory board of Boston Scientific and Medtronic and has received honoraria from Boston Scientific, Medtronic, Abbott, Teva and Ipsen.

\section{Author contributions}

GRW: Data curation; Formal analysis; Investigation; Methodology; Visualization; Roles/Writing original draft; Writing - review \&editing

JR: Methodology; Visualization; Roles/Writing - original draft; Writing - review \&editing

CB: Investigation; Writing - review \&editing

ALM: Investigation; Writing - review \&editing

EG: Investigation

MPN: Supervision; Validation

HS: Project administration; Resources

KW: Data curation; Software; Resources

LJP: Conceptualization, Data curation; Formal analysis; Methodology; Software; Resources; Roles/Writing - original draft; Writing - review \&editing

AAK: Conceptualization; Methodology; Supervision; Validation; Writing - review \&editing; Funding acquisition

\section{Acknowledgement}

JR is funded by a research project to AAK by the Deutsche Forschungsgemeinschaft (DFG, German Research Foundation) under Germany's Excellence Strategy - EXC-2049 - 39068808.

\section{Role of the funding source}

Boston Scientific provided financial support for the conduct of the research, but not for the preparation of the article and will not pay the article publication charges. Boston Scientific participated in the study design, prepared formal documents and provided (e)CRFs for the collection of data. Furthermore, Boston Scientific drafted the 'Subjects \& Methods' section and provided review for the statistical analysis and the manuscript. All data analysis was performed by GRW and JR 
independently from Boston Scientific. A review board of Boston Scientific approved the decision to submit the article for publication but did not make any suggestions for changes to the manuscript.

\section{Author statement}

With the submission of the manuscript, we would like to undertake that:

- $\quad$ All authors of this paper have directly participated in planning, execution, or analysis.

- $\quad$ All authors of this paper have read and approved the final version submitted.

- The contents of this manuscript have not been copyrighted or published previously.

- The contents of this manuscript are not now under consideration for publication elsewhere.

- The contents of this manuscript will not be copyrighted, submitted, or published elsewhere, while acceptance by the journal is under consideration;

- $\quad$ There are no directly related manuscripts or abstracts, published or unpublished, by any authors of this paper.

Furthermore, we declare that the study in this manuscript has been carried out according to ICH-GCP standards, that we received approval from both local Institutional Review Boards in the two study centers and that the experiments were done or in accord with the Helsinki Declaration of 1975.

Financial disclosures are included in the manuscript. As stated also in the manuscript the sponsor of the study, Boston Scientific provided financial support for the conduct of the research, but not for preparation of the article and will not pay the article publication charges. Boston Scientific participated in the study design, prepared formal documents and provided (e)CRFs for the collection of data. Furthermore, Boston Scientific drafted the 'Subjects \& Methods' Section and provided review for the statistical analysis and the manuscript. All data analysis was performed by the authors Gregor R. Wenzel and Jan Roediger independently from Boston Scientific. A review board of Boston Scientific approved the decision to submit the article for publication, but did not make any suggestions for changes to the manuscript. 